OPEN ACCESS

Edited by:

Alistair Dove,

Georgia Aquarium, United States

Reviewed by:

Natascha Wosnick,

Federal University of Paraná, Brazil

Christine Dudgeon,

The University of Queensland,

Australia

*Correspondence:

Taketeru Tomita

t-tomita@okichura.jp

Specialty section:

This article was submitted to

Marine Conservation

and Sustainability,

a section of the journal

Frontiers in Marine Science

Received: 30 November 2021

Accepted: 07 February 2022

Published: 02 March 2022

Citation:

Tomita T, Toda M, Murakumo K, Kaneko A, Yano N, Nakamura M and Sato K (2022) Five-Month Incubation of Viviparous Deep-Water Shark

Embryos in Artificial Uterine Fluid.

Front. Mar. Sci. 9:825354.

doi: 10.3389/fmars.2022.825354

\section{Five-Month Incubation of Viviparous Deep-Water Shark Embryos in Artificial Uterine Fluid}

\author{
Taketeru Tomita1,2*, Minoru Toda², Kiyomi Murakumo², Atsushi Kaneko², Nagisa Yano², \\ Masaru Nakamura ${ }^{1}$ and Keiichi Sato ${ }^{1,2}$ \\ ${ }^{1}$ Okinawa Churashima Research Center, Okinawa Churashima Foundation, Okinawa, Japan, ${ }^{2}$ Okinawa Churaumi Aquarium, \\ Okinawa Churashima Foundation, Okinawa, Japan
}

In this study, we developed an extra-uterine life-support system (artificial uterus) for viviparous elasmobranchs. Using this system, we maintained two embryonic specimens of the slendertail lantern shark (Etmopterus molleri) for approximately 5 months, the longest published record of the captive maintenance of the embryos of this species. This system is characterized by the use of a urea-containing artificial incubation fluid, of which the salinity and osmotic pressure are approximately equivalent to those of shark blood plasma. We hypothesized that this fluid reduces the salinity gradient between embryonic blood plasma and the environmental fluid, which thereby contributes to countering the low osmoregulatory capacity of viviparous shark embryos. However, the incubated specimens subsequently died during the process of seawater adaptation following "artificial birth." Accordingly, information pertaining to uterine chemical dynamics throughout the gestation period is necessary for the design of a more effective incubation system. This technique will be relevant and may find application in conservation breeding at public aquaria in the future.

Keywords: artificial uterus, bioluminescence, elasmobranch, Etmopterus molleri, extra-uterine life-support system

\section{INTRODUCTION}

An extra-uterine life support system, or artificial uterus, represents a novel option for the medical treatment of premature fetuses. By supporting fetal respiration and nutrition, such systems facilitate fetal development outside the uterus. In a pilot study on this technology, Partridge et al. (2017) used a system that supplies oxygen and nutrients to fetal blood through a plastic tube connected to the umbilical cord, and succeeded in maintaining lamb fetuses in a plastic bag ("biobag") filled with artificial amniotic fluid for a maximum of 28 days. Such artificial uteruses have applications not only for medical treatment in human hospitals, but also in public zoos and aquaria. In recent years, zoos and aquaria are expected to function as nature conservation facilities, and captive breeding in particular is probably the most direct way to contribute to increases in wild populations (Rabb and Saunders, 2005). However, captive breeding techniques are not yet well established for many vertebrate species including elasmobranchs (sharks, skates, and rays). More than $80 \%$ of the 387 species of elasmobranchs listed as threatened species by the IUCN Red List are viviparous, and the technology to maintain viviparous embryos that are born prematurely or extracted alive from the dead female is necessary to establish conservation breeding technologies. 
In this paper, we report the development of an artificial uterus designed specifically for elasmobranchs, and describe its preliminary application for sustaining viviparous shark embryos. Using this system, we maintained embryos of the slendertail lantern shark (Etmopterus molleri) for longer than 5 months until "birth," the longest reliable record of the maintenance of viviparous elasmobranch embryos in captivity. The slendertail lantern shark is a relatively small (up to $46 \mathrm{~cm}$ in total length) deep-water shark that is distributed in the West Pacific (Compagno, 1984). The genus Etmopterus is particularly noted for its bioluminescence capacity, which is attributed to the large number of specific photogenetic organs (photophores) distributed across the body surface that emit a blue-green light generated via biochemical reactions (e.g., Duchatelet et al., 2019). Etmopterus has several advantages in the development of an artificial uterus in elasmobranchs. First, this genus is commonly distributed around Okinawa and is available as bycatch from local fishermen. Second, embryonic development in Etmopterus is largely sustained by its own yolk, with little nutritional input from the mother to the embryo (Cotton et al., 2015). This is in contrast to some viviparous elasmobranchs, such as lamniform sharks and myliobatiform stingrays, in which large quantities of nutrients are supplied from the mother to the embryo (Musick and Ellis, 2005). Thus, the high degree of independence of embryonic nutrition in Etmopterus allows for the development of the simplest artificial uterus systems and can provide a technical basis for more complex systems involving mechanisms to supply nutrients to the embryo. In the past, the husbandry team at Okinawa Churaumi Aquarium have strived to maintain these sharks, both adults and embryos, in cold filtered seawater. However, despite more than 20 years of attempting, they have yet to succeed in maintaining these sharks in captivity for more than a week (MT, pers. obs.).

\section{MATERIALS AND METHODS}

\section{Artificial Uterus}

The core system of the newly designed artificial uterus comprises three elements, namely, the main chamber, reserve tank, and filtering system (Figure 1). The main chamber, in which the embryos are incubated, is cylindrical in shape, with a diameter and depth of 50 and $60 \mathrm{~cm}$, respectively. The incubation fluid enters the chamber from bottom, flows upward at a steady rate of $10 \mathrm{~L} / \mathrm{min}$, and is expelled via the top of the chamber. Two $500-\mathrm{mL}$ plastic jars, each containing a single embryo, are place at middepth in the chamber. The mouth of these jars is covered with a plastic net (mesh size $=1.5 \mathrm{~mm}$ ), which facilitates free exchange of fluid between the inside and outside of the jar.

The fluid that is expelled from the main chamber is channeled into the reserve tank, wherein it is aerated and pumped into the filtering system, flowing through 10.0, 2.0, and $0.65 \mu \mathrm{m}$-mesh filters. Having been filtered, the fluid is sterilized by exposing to UV light (UVF1000; IWAKI Co., Ltd., Tokyo) and thereafter returned to the main chamber. The temperature of the fluid in the artificial uterus is maintained at $13^{\circ} \mathrm{C}$.

The supply tank functions as a peripheral system that supplies fresh incubation fluid to the core system of the artificial uterus.
The fluid in this tank is maintained at a temperature of $12^{\circ} \mathrm{C}$ and is periodically delivered to the reserve tank at a rate of $30-$ $50 \mathrm{~mL} / \mathrm{min}$.

\section{Artificial Uterine Fluid}

For the purposes of the present study, we used a fluid that is isotonic to shark blood plasma (herein referred to as "artificial uterine fluid") for embryonic incubation. This fluid was prepared by dissolving $3.5 \mathrm{~kg}$ urea to $46 \mathrm{~L}$ of chlorine-free tap water, which was made up to a final volume of $100 \mathrm{~L}$ with filtered seawater. The salinity and osmotic pressure of this fluid (the chemical composition of which is listed in Table 1) are approximately equivalent to those in shark blood plasma. The salinity of the fluid was established based on reference to the sodium concentration in the blood plasma in Triakis scyllium (Takei, 2012). The amount of dissolved urea was determined based on the sum of the molarities of blood urea and trimethylamine $\mathrm{N}$-oxide (TMAO), the primary and secondary major osmotic solutes in shark blood plasma, respectively. The molarity of blood urea was based on the value determined for T. scyllium (Takei, 2012), whereas the molarity of blood TMAO, the value of which in T. scyllium has not been determined, was based on the value in Rhincodon typus (KM, unpublished data).

\section{The Maintenance of Embryos in Captivity}

A single pregnant slendertail lantern shark (Etmopterus molleri) was obtained from local fishermen on the main island of Okinawa, Japan, who captured the specimen during hook-andline fishing. Following capture, the shark had been maintained in a cooler box filled with seawater in the fishing boat, but died during the 6-h return trip and was donated to the Okinawa Churaumi Aquarium (Okinawa) for scientific purposes. Ultrasound examination of this specimen revealed that the uterus contained a total of six living embryos, which were recovered using a scalpel and forceps. Two randomly selected embryos (specimens 1 and 2) were subsequently placed in the aforementioned artificial uterus set up at the aquarium (day 1 of embryonic incubation) (Figure 2), whereas the remaining four embryos (specimens 3-6) were placed in small aquaria filled with $13^{\circ} \mathrm{C}$ filtered seawater.

Embryonic incubation in the artificial uterus was performed in two steps. In step 1, the embryos were incubated solely in the artificial uterine fluid, with $120 \mathrm{~L}$ of fresh incubation fluid being introduced into the artificial uterus approximately once per week to flush out the accumulated ammonia. Step 2 constitutes a preparatory phase initiated prior to birth. This step is similar to step 1, although differs with respect to the periodical exposure of embryos to seawater. For this process, $110 \mathrm{~L}$ of seawater was introduced into the artificial uterus once a week. On the day after each seawater addition, the same volume of fresh artificial uterine fluid was introduced into the artificial uterus to maintain the requisite sodium and urea concentrations in the main chamber.

The embryos were released from the plastic jars when the external yolk was fully retracted. This process facilitates fetal transition from a state of highly restricted movement in a confined space to a state of more free movement. We defined 

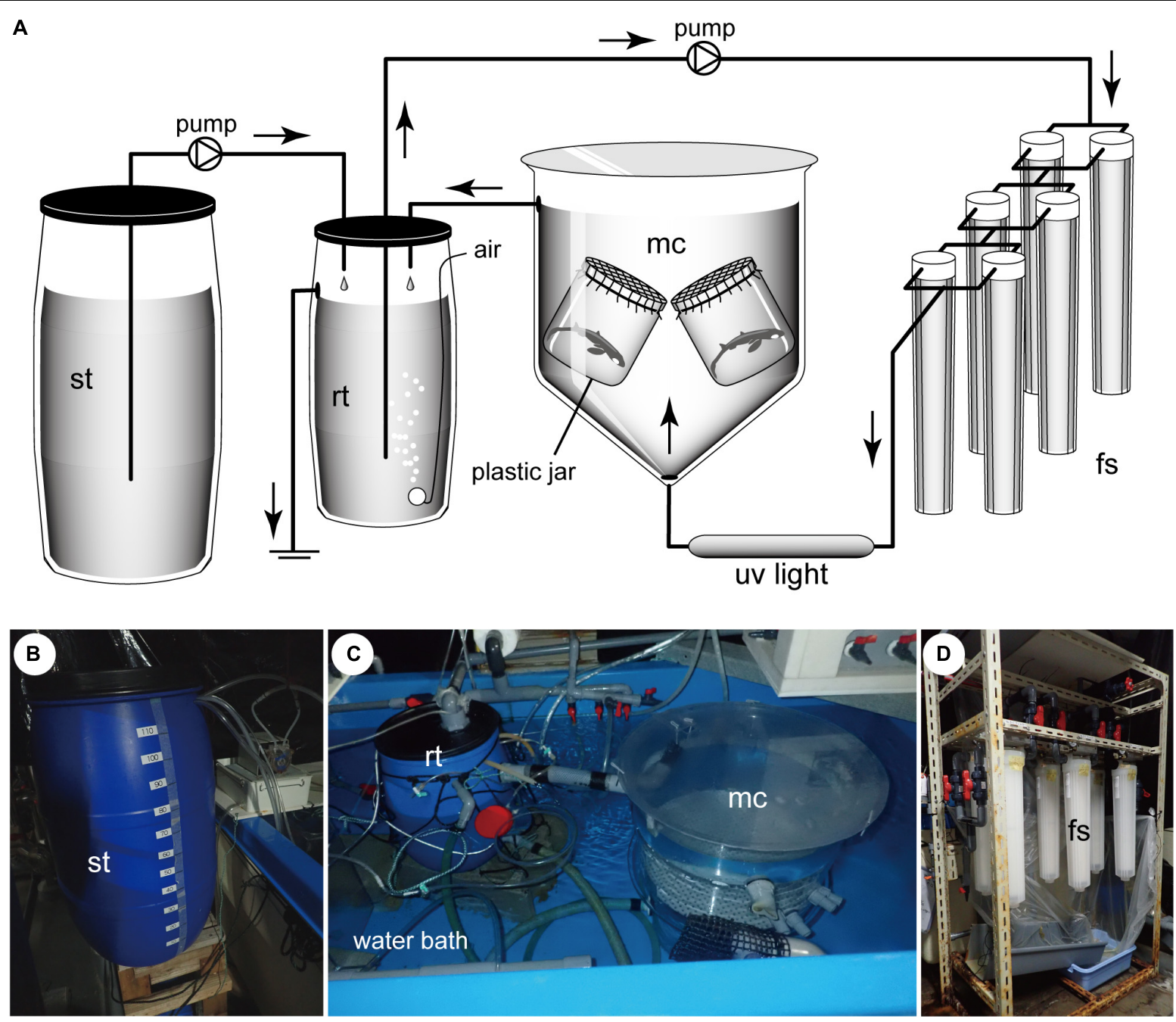

FIGURE 1 | (A) The circuits and components of the artificial uterus developed in this study, consisting of a main chamber (mc), reserve tank (rt), filtering system (fs), and supply tank (st). (B-D) Photographs of components of the artificial uterus shown in (A). Main chamber and reserve tank are placed in a water bath to maintain an internal temperature of $13^{\circ} \mathrm{C}$.

this process as "artificial birth" and the day on which this event occurred was defined as day 1 after birth. Immediately after this operation, specimen 1 was moved to a seawater tank located externally to the artificial uterus. This tank is cylindrical in shape, with a diameter of $100 \mathrm{~cm}$ and depth of 100 and $60 \mathrm{~cm}$, respectively, and was filled with $1,000 \mathrm{~L}$ of seawater. Water temperature was maintained at $12^{\circ} \mathrm{C}$. In contrast, specimen 2 was retained in the main chamber of the artificial uterus, and the fluid within the chamber was gradually replaced with seawater over the course of the 2 -week period after birth.

\section{Monitoring of Water Chemistry and Embryonic Development}

The water temperature in the main chamber was measured daily using a digital thermometer. Water chemistry (sodium, urea, and ammonia) was monitored using a DRI-CHEM $7000 \mathrm{~V}$ automated clinical chemistry analyzer (Fujifilm Co., Tokyo,
TABLE 1 | Chemical measurements and osmotic pressure of artificial uterine fluid $(n=17)$.

\begin{tabular}{lccc}
\hline Measure & Unit & Average & SD \\
\hline Sodium $(\mathrm{Na})$ & $\mathrm{mmol} / \mathrm{L}$ & 291.5 & 6.2 \\
Potassium $(\mathrm{K})$ & $\mathrm{mmol} / \mathrm{L}$ & 5.7 & 0.2 \\
Chlorine $(\mathrm{Cl})$ & $\mathrm{mmol} / \mathrm{L}$ & 263.8 & 6.0 \\
Ammonia $\left(\mathrm{NH}_{3}\right)$ & $\mu \mathrm{mol} / \mathrm{L}$ & 30.3 & 18.0 \\
Urea $\left(\mathrm{CH}_{4} \mathrm{~N}_{2} \mathrm{O}\right)$ & $\mathrm{mmol} / \mathrm{L}$ & 540.2 & 15.6 \\
Osmotic pressure & $\mathrm{mmol} / \mathrm{kg}$ & 1085.4 & 32.2 \\
\hline
\end{tabular}

Japan). The abundance of bacteria in the artificial uterine fluid was quantified using an Uricult E culture system (Sekisui Medical Co., Tokyo, Japan).

The condition of embryos was visually assessed twice daily (at 10:00 and 17:00) by confirming the presence of respiratory behavior (e.g., rhythmical gill flap motion and opening/closing 


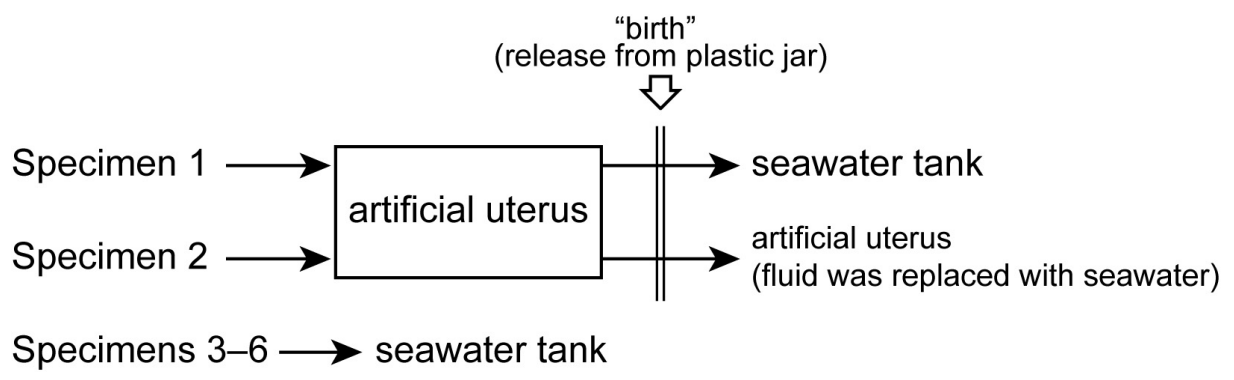

FIGURE 2 | An overview of the incubation experiment using lantern shark embryos.

of spiracle) and reaction to red light (e.g., body undulation and head reorientation), and were also recorded using a GoPro HERO 5 camera (GoPro Inc., San Mateo, United States) once or twice weekly. To minimize potential stress to the embryos, the duration of each observation period was limited to a maximum of $5 \mathrm{~min}$. To determine temporal changes in the size of the external yolk sac, we calculated the relative external yolk sac size (r) using the following equation:

$$
r=\frac{y s w}{h d l}
$$

where $y s w$ is the maximum width of the external yolk sac and $h d l$ is the head length (Figure 3). Both values were measured from the pixels of still images captured on GoPro video footage, using ImageJ software.

\section{RESULTS}

\section{Embryonic Incubation}

Until birth, embryo specimens 1 and 2 were maintained in the artificial uterus for 146 and 160 days, respectively. Throughout these periods, the total length of the specimens increased from approximately 10 to $15 \mathrm{~cm}$. The specimens were initially characterized by the presence of external gill filaments; however, these filaments had completely disappeared by 60 days after the onset of incubation. The retrieved specimens also initially had an ellipsoid external yolk sac, the widths of which were approximately $1 \mathrm{~cm}$ (approximately $40-60 \%$ of the head length; Figure 3). During the course of incubation, the external yolk sac decreased in size and had become fully retracted at approximately 150 days after incubation onset (Figure 3). During the first month under the incubation conditions, the embryos remained relatively inactive, with activity being mainly restricted to respiratory motion. Thereafter, however, the embryos became more active, showing a strong swimming motion in response to external light stimuli. On the basis of the external morphological features of the specimens, the embryonic development observed in the present study was assessed to correspond to developmental stages 32-34 of Scyliorhinus canicula (Ballard et al., 1993).

Subsequent to birth, specimens 1 and 2 died on days 4 and 25 post-birth, respectively, which we suspect may have been attributable to a failure to adapt to the seawater environment. During the post-birth period, specimen 2 was observed to consume $0.1 \mathrm{~g}$ sakura shrimp (Lucensosergia lucens) meat on the 2nd day and two $0.15 \mathrm{~g}$ pieces of chub mackerel (Scomber japonicus) meat on the 9th day. For specimens 1 and 2, the total duration of maintenance in captivity was 150 and 184 days, respectively, which were considerably longer than that of the sibling embryos (specimens 3-6), which were maintained in seawater and survived for between 5 and 7 days (average $=6.3$ days).

\section{Changes in Water Chemistry}

The changes in sodium, urea, and ammonia concentrations in the artificial uterus are shown in Figure 4. In step 1 of the incubation period, the concentrations of sodium and urea remained relatively stable at approximately 300 and $540 \mathrm{mmol} / \mathrm{L}$, respectively. Contrastingly, during step 2, the introduction of seawater induced 10 corresponding spike-like fluctuations in the sodium and urea concentrations. Unlike the temporal patterns in sodium and urea concentrations, there was no clear difference between steps 1 and 2 with respect to ammonia concentrations. The frequent introduction of fresh artificial uterine fluid or seawater did, nevertheless, contribute to a long-term reduction in ammonia concentrations until the time of birth. The salinity of the artificial uterine fluid was calculated to be approximately $19.2 \%$, which was obtained based on the average value of seawater measured at the aquarium.

\section{Detection of Bacteria in the Artificial Uterus}

Throughout the period of incubation, the number of bacteria in the incubation fluid within the main chamber was maintained at levels below the limit of detection.

\section{DISCUSSION}

The 160-day incubation achieved in this study is not only the longest record of maintaining embryonic lantern sharks in captivity but also among the longest periods of ex situ maintenance of viviparous elasmobranchs. To the best of our knowledge, the first documentation of the captive maintenance 


\section{A}

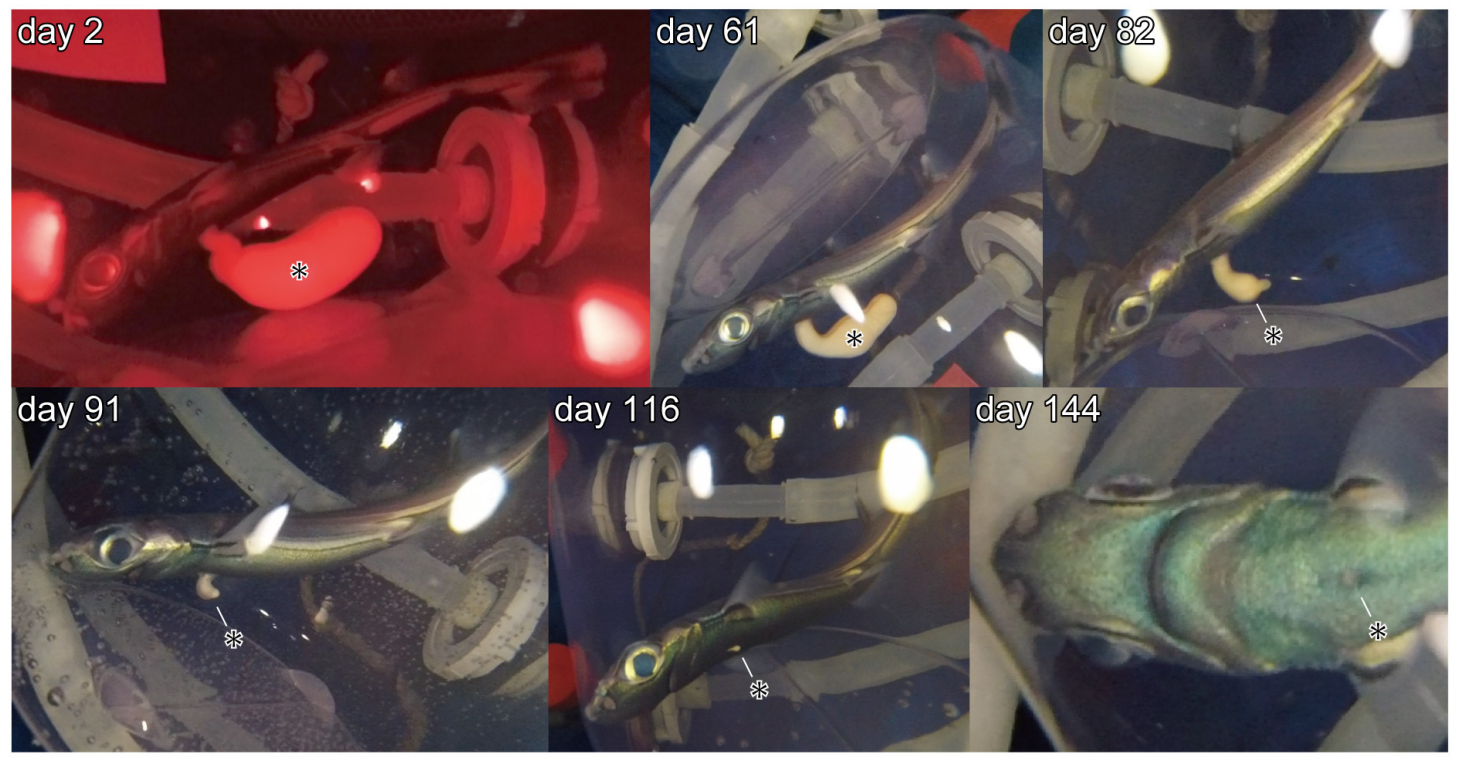

B

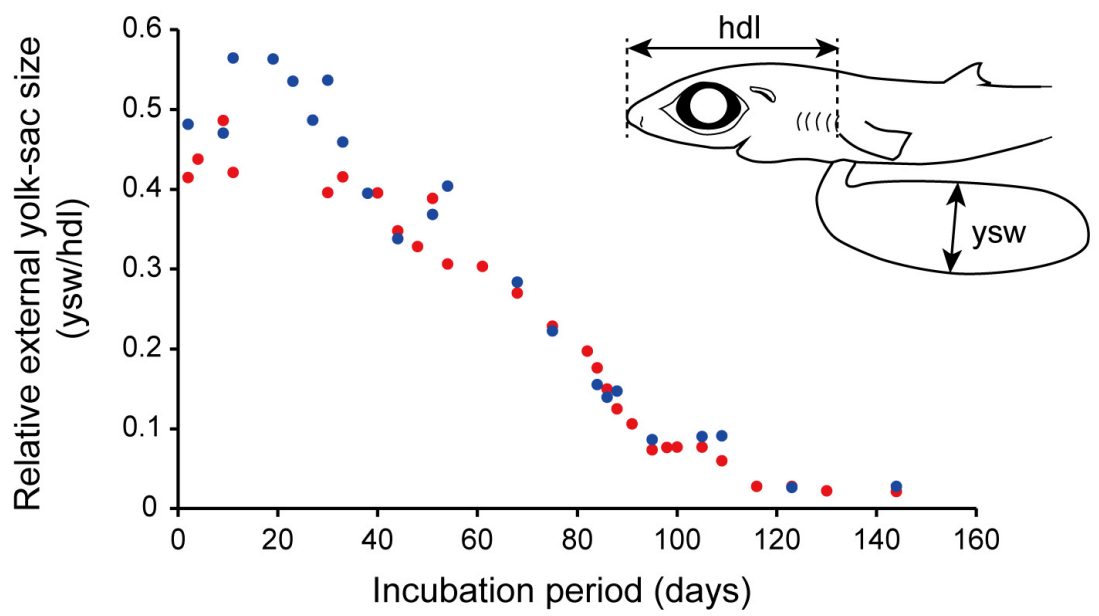

FIGURE 3 | An embryo specimen incubated in the artificial uterus. (A) Temporal changes in embryo external morphology, showing a reduction in the size of the external yolk-sac (*). (B) Changes in relative external yolk-sac size in specimen 1 (red) and specimen 2 (blue). The upper right-hand illustration in (B) represents the anterior half of the body of a lantern shark embryo, showing the locations of measurements used for calculating relative external yolk-sac size. hdl, head length; ysw, external yolk-sac width.

of embryonic viviparous elasmobranchs was that reported by Gilbert (1959), who maintained a late-term embryo of Squalus acanthias in seawater for 40 days. Similarly, Evans et al. (1982) maintained mid- to late-term embryos of $S$. acanthias in seawater for 7 days. More recently, Otway and Ellis (2012) have reported the maintenance of late-term embryos of Orectolobus ornatus for 18 days in seawater until "artificial parturition." However, details of the longest recorded maintenance of elasmobranch embryos in captivity, albeit unpublished in the scientific literature, can be found on the webpage of the joint project between the Marine Science Museum Social Education Center, Tokai University (Shizuoka, Japan), and Aquamarine Fukushima (Fukushima, Japan), which indicate that embryonic
Chlamydoselachus anguineus were sustained ex situ for 361 days (accessed on 2021.6.11). ${ }^{1}$

Given our findings that lantern shark embryos appear to be unable to survive in seawater for more than a week, it would appear likely that the artificial uterine fluid used in the present study played an essential role in prolonging embryo survival under captive conditions, although the underlying mechanisms remain to be determined. In this regard, however, we hypothesize that a small salinity gradient between blood plasma and environmental fluids is essential for maintaining a stable embryonic blood chemistry. This conjecture is based on the

\footnotetext{
${ }^{1}$ https://www.umi.muse-tokai.jp/news/single.php?id=117
} 


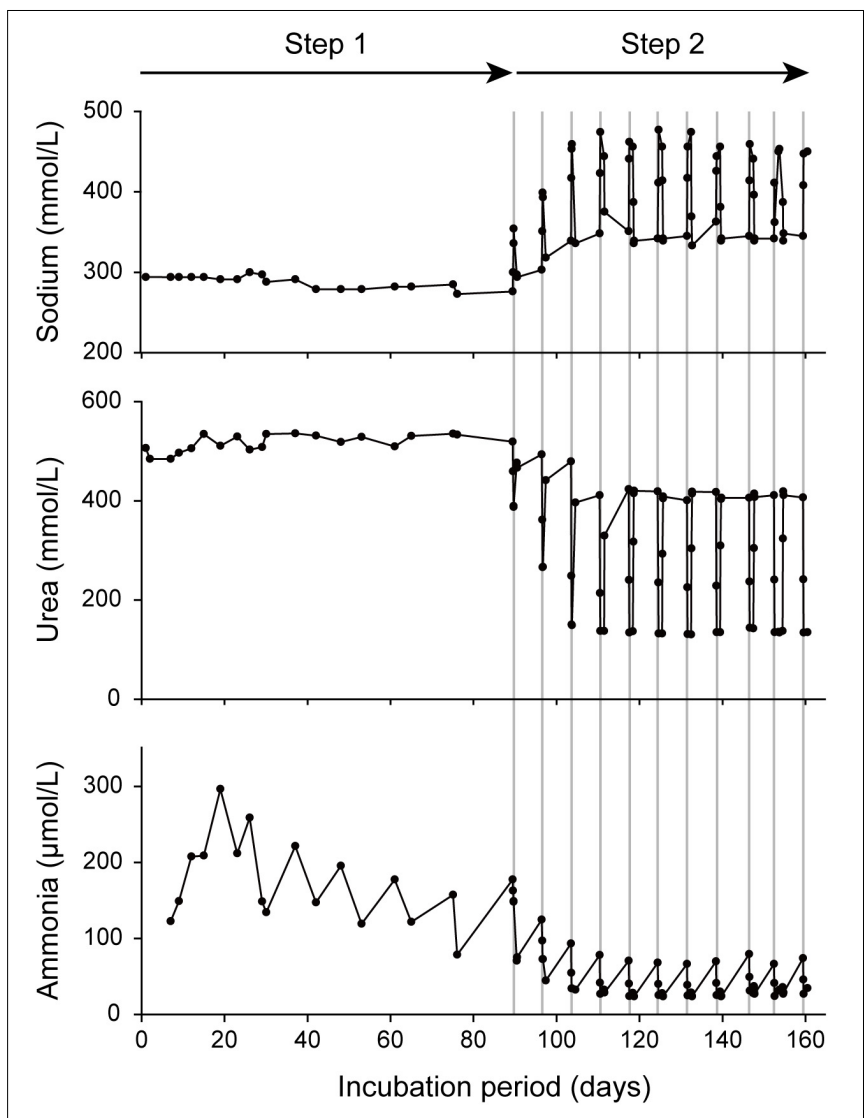

FIGURE 4 | Temporal change in the chemical composition (sodium, urea, and ammonia) of the incubation fluid prior to artificial "birth." In the initial period of incubation (step 1), the specimens were maintained exclusively in the artificial uterine fluid, whereas in the latter phase of incubation (step 2), the specimens were periodically exposed to seawater (the durations of seawater exposure are shown in area denoted by gray lines).

observations reported by Evans et al. (1982), who placed mid- and late-term embryos of viviparous Squalus acanthias in seawater and monitored the ensuing changes in blood chemistry, thereby revealing that the salinity of embryonic blood plasma was initially half the value of full-strength seawater, as in adult sharks, but shifted to a level close to that of seawater within a few days. These observations thus tend to indicate that in a high-salinity environment, the osmoregulatory capacity of viviparous shark embryos is not sufficiently strong to maintain blood salinity at an appropriate level. Our hypothesis that artificial uterine fluid contributes to maintaining embryonic osmoregulation could be further evaluated by demonstrating that the activity of potential embryonic osmoregulatory organs, such as the kidney, rectal gland, and external yolk sac, is maintained at a low level when the embryo is maintained in the artificial uterine fluid. Such information can be obtained based on immunohistochemical and gene expression analyses, as demonstrated by Takagi et al. (2014) in the case of embryos of the oviparous holocephalan Callorhinchus milii.

The remaining technical challenge is how to safely acclimate the incubated specimens to the seawater environment following "artificial birth." We attempted to accomplish this process by periodically exposing the specimens to seawater prior to "artificial birth," although this approach eventually proved unsuccessful. This gradual acclimation process was designed to mimic the mechanisms whereby some viviparous sharks, including Squalus acanthias and Orectolobus ornatus, periodically replace uterine fluid with external seawater during late gestation (referred to as uterine flushing by Ellis and Otway, 2011). In S. acanthias, the chemical composition of the uterine fluid is known to change through ontogeny. In early gestation, the ionic composition of the uterine fluid is similar to that of the maternal plasma, whereas in late gestation, it becomes closer to that of seawater (Evans et al., 1982; Kormanik and Evans, 1986; Kormanik, 1988, 1993). Acquisition of similar data for the uterine fluid in lantern sharks is accordingly considered essential for designing an appropriate pre-adaptation process for these species.

The development of an effective artificial uterus would be useful in the study of currently uncharacterized aspects of lantern shark ecology. In this regard, a feature of particular interest in the ecologies of this group of sharks is their ability to bioluminescence, which has been a subject of numerous studies that have focused on its mechanism. For example, it has been suggested that the bioluminescence of lantern sharks is not bacterial in origin, but is instead mediated via an inherent biochemical reaction, referred to as the luciferinluciferase reaction (e.g., Duchatelet et al., 2019). One of the mysteries regarding this mechanism concerns the origin of luciferin. It has traditionally been assumed that lantern shark luciferin, although as yet unidentified, is an endogenous product of shark metabolism. However, Mizuno et al. (2021) have recently claimed that lantern shark luciferin is instead derived from the food they consume. This hypothesis is, nevertheless, still under debate, given that coelenterazine, a luciferin that Mizuno et al. (2021) believe to be lantern shark luciferin, has hitherto not been detected in lantern shark photophores (Renwart and Mallefet, 2013). As there is no likelihood that lantern shark specimens incubated in an artificial uterus would receive external inputs of coelenterazine, either from environmental fluid or the maternal body, we could establish the validity of Mizuno et al.'s (2021) hypothesis by (1) confirming whether or not these specimens emit light without an external input of coelenterazine and, if not, (2) confirming whether the oral administration of coelenterazine promotes the subsequent development of bioluminescence.

In summary, we developed a novel artificial uterus system for viviparous sharks and showed that lantern shark embryo development can be completed using this system. In the context of designing future zoos and aquariums, technology for animal conservation will become increasingly important (Rabb and Saunders, 2005). This technique of ex situ embryonic incubation contributes to animal conservation in the following ways: First by increasing the success rate of captive breeding programs and thereby reducing the burden on nature to maintain aquarium exhibits. Second, the technology can be applied to conservation breeding at public aquaria and aid in the recovery of wild animal populations, especially those of endangered species. Development of similar techniques applicable to a wider range 
of taxa and the rapid sharing of such information are vital to achieving this objective.

\section{DATA AVAILABILITY STATEMENT}

The original contributions presented in the study are included in the article/supplementary material, further inquiries can be directed to the corresponding author/s.

\section{ETHICS STATEMENT}

Ethical review and approval was not required for the animal study because though the maintenance and handling of fish in this study were conducted in strict accordance with the guidelines for animal experiments of the Okinawa Churashima Foundation, with the same consideration for animal care and welfare as that for "higher" vertebrates (reptiles, birds, and mammals), as the guidelines stipulated, the approval from the Institutional Animal Care and Use Committee of Okinawa Churashima Foundation, required for higher vertebrates, is waived for "lower" vertebrates including fishes.

\section{REFERENCES}

Ballard, W. W., Mellinger, J., and Lechenault, H. (1993). A series of normal stages for development of Scyliorhinus canicula, the lesser spotted dogfish (Chondrichthyes: Scyliorhinidae). J. Exp. Zool. 267, 318-336.

Compagno, L. J. V. (1984). Sharks of the world. An annotated and illustrated catalogue of shark species known to date. FAO Fish. Synop. 125:249.

Cotton, C. F., Grubbs, R. D., Dyb, J. E., Fossen, I., and Musick, J. A. (2015). Reproduction and embryonic development in two species of squaliform sharks, Centrophorus granulosus and Etmopterus princeps: evidence of matrotrophy? Deep Sea Res. 115, 41-54.

Duchatelet, L., Delroisse, J., Flammang, P., Mahillon, J., and Mallefet, J. (2019). Etmopterus spinax, the velvet belly lanternshark, does not use bacterial luminescence. Acta Histochem. 121, 516-521. doi: 10.1016/j.acthis.2019. 04.010

Ellis, M. T., and Otway, N. M. (2011). Uterine fluid composition of the dwarf ornate wobbegong shark (Orectolobus ornatus) during gestation. Mar. Freshw. Res. 62, 576-582.

Evans, D. H., Oikari, A., Kormanik, G. A., and Mansberger, L. (1982). Osmoregulation by the prenatal spiny dogfish, Squalus acanthias. J. Exp. Biol. 101, 295-305.

Gilbert, P. W. (1959). The ability of yolk-sac dogfish pups to survive outside the uterus. Bull.Mt. Desert Isl. Biol. Lab. 1959:68.

Kormanik, G. A. (1988). Time course of the establishment of uterine seawater conditions in late-term pregnant spiny dogfish (Squalus acanthias). J. Exp. Biol. 137, 443-456.

Kormanik, G. A. (1993). Ionic and osmotic environment of developing elasmobranch embryos. Environ. Biol. Fishes. 38, 233-240.

Kormanik, G. A., and Evans, D. H. (1986). The acid-base status of prenatal pups of the dogfish. J. Exp. Biol. 125, 173-179.

Mizuno, G., Yano, D., Paitio, J., Endo, H., and Oba, Y. (2021). Etmopterus lantern sharks use coelenterazine as the substrate for their luciferin-luciferase bioluminescence system. Biochem. Biophys. Res. Commun. 577, 139-145. doi: 10.1016/j.bbrc.2021.09.007

Musick, J. A., and Ellis, J. K. (2005). "Reproductive evolution of chondrichthyans," in Reproductive Biology and Phylogeny of Chondrichthyes, ed. W. C. Hamlett (Boca Raton: CRC Press), 45-79.

\section{AUTHOR CONTRIBUTIONS}

TT was responsible for experimental design, experimental operation, data analysis, and article writing. MT was responsible for experimental design, experimental operation, and data analysis. KM, AK, and NY were responsible for experimental operation, instrument operation, and data analysis. KS was responsible for experimental design and overall thinking. All authors contributed to the article and approved the submitted version.

\section{FUNDING}

This work was supported by the JSPS KAKENHI (Grant Nos. 17K19334 and 19K04064).

\section{ACKNOWLEDGMENTS}

We thank the staff of the Okinawa Churaumi Aquarium for providing data and assistance.

Otway, N. M, and Ellis, M. T. (2012). Construction and test of an artificial uterus for ex situ development of shark embryos. Zoo Biol. 31, 197-205. doi: 10.1002/ zoo. 20422

Partridge, E. A., Davey, M. G., Hornick, M. A., McGovern, P. E., Mejaddam, A. Y., Vrecenak, J. D., et al. (2017). An extra-uterine system to physiologically support the extreme premature lamb. Nat. Commun. 8:15112.

Rabb, G. B., and Saunders, C. D. (2005). The future of zoos and aquariums: conservation and caring. Int. Zoo Year Book 39, 1-26. doi: 10.1111/j.1748-1090. 2005.tb00001.x

Renwart, M., Mallefet, J. (2013). First study of the chemistry of the luminous system in a deep-sea shark, Etmopterus spinax Linnaeus, 1758. J. Exp. Mar. Biol. Ecol. 448, 214-219.

Takagi, W., Kajimura, M., Tanaka, H., Hasegawa, K., Bell, J. D., Toop, T., et al. (2014). Urea-based osmoregulation in the developing embryo of oviparous cartilaginous fish (Callorhinchus milii): contribution of the extraembryonic yolk sac during the early developmental period. J. Exp. Biol. 217, 1353-1362. doi: $10.1242 /$ jeb.094649

Takei, Y. (2012). Mechanism of osmoregulation in fishes: adaptation to seawater environment. Proc. Symp. Salt Sci. 2012, 1-7. (in Japanese).

Conflict of Interest: The authors declare that the research was conducted in the absence of any commercial or financial relationships that could be construed as a potential conflict of interest.

Publisher's Note: All claims expressed in this article are solely those of the authors and do not necessarily represent those of their affiliated organizations, or those of the publisher, the editors and the reviewers. Any product that may be evaluated in this article, or claim that may be made by its manufacturer, is not guaranteed or endorsed by the publisher.

Copyright (C) 2022 Tomita, Toda, Murakumo, Kaneko, Yano, Nakamura and Sato. This is an open-access article distributed under the terms of the Creative Commons Attribution License (CC BY). The use, distribution or reproduction in other forums is permitted, provided the original author(s) and the copyright owner(s) are credited and that the original publication in this journal is cited, in accordance with accepted academic practice. No use, distribution or reproduction is permitted which does not comply with these terms. 